\title{
Screening for Oral Cancer: A Review
}

\section{ISHAN PRABHAKAR}

Oral cancer is a common disease which affects individuals of mainly the older age group. If diagnosed at later stages, the prognosis is poor. In this review, the various techniques for diagnosis of oral cancer such as screening of the patient by conventional oral examination, supravital staining, visual adjunctive aids, biopsy etc. have been discussed. The traditional treatments include surgery, chemotherapy and radiotherapy. Newer modalities include gene therapy, immunotherapy, etc. have proven to be successful to some extent. Thorough knowledge of the disease and timely diagnosis by the dental professional can prevent metastasis.

KEYWORDS: Oral Cancer, Screening, Staining

\section{INTRODUCTION}

It is a real challenge for an oral health professional to provide satisfactory dental care to patients. Most oral health conditions can be prevented or treated successfully with education and regular dental and dental hygiene care. Unfortunately, the one oral disease that can be life-threatening is the disease that is often overlooked with respect to emphasis on prevention and early detection in clinical practice settings.

Research states that Oral cancer is the $6^{\text {th }}$ most common malignancy in the world. ${ }^{1}$ It is estimated that over 640,000 new cases will be identified worldwide on a yearly basis.

The prognosis for oral and pharyngeal cancer varies depending upon when it is identified. Approximately 83 percent of patients with localized lesions survive beyond five years. ${ }^{2}$ Prognosis is significantly worse when oral cancer has metastasized resulting in a 28 percent survival rate. ${ }^{3}$ Oral cancer has a high risk of producing second primary tumors. If a patient survives the initial diagnosis and treatment, they still have up to a 20 time higher risk of developing a second cancer. ${ }^{4}$

These statistics can be improved with emphasis on prevention and early detection. Unfortunately, oral and pharyngeal cancer is routinely discovered at a later stage in development when the cancer has already metastasized. Part of the problem is that early cancer is painless and not easily recognized by the patient. The other contributing element is that dental practitioners are not placing the same level of importance on early detection of oral cancer as they place on the prevention and treatment of caries and periodontal disease. ${ }^{6}$ Therefore, this paper highlight key elements of oral cancer as well as current approaches used for early detection.

\section{RISK FACTORS/ETIOLOGY OF ORAL CANCER}

- History of tobacco use (all forms)

- Alcohol consumption

- Sexual history (multiple sex partners, unprotected sex, exposure to HPV)

- Exposure to ultraviolet light

- Exposure to ionizing radiation, arsenic or industrial chemicals

- Chronic irritation (poor oral hygiene, poor restorative dentistry)

- Pre-existing scars and burns

- Family history of cancer

- Personal history of cancer

- Age $>40$ years

- Mucosal diseases (iron deficiency associated with Plummer-Vinson Syndrome, lichen planus)

- Immune system suppression

- Gender

- Race

\section{WARNING SIGNS OF ORAL CANCER}

- A lump or thickening in the oral soft tissues

- Soreness or difficulty in chewing or swallowing

- Ear pain

- Difficulty moving the jaw or tongue

- Hoarseness

- Numbness of the tongue or other areas of the mouth 
- Swelling of the jaw that causes dentures to fit poorly or become uncomfortable

- Repeated bleeding from the mouth or throat

- Red, white, or discolored lesions in the mouth or on the lips

\section{SIGNS AND SYMPTOMS OF ORAL CANCER}

- Intraoral swelling on lips or neck

- Crust on lip

- Bleeding

- Rough spot

- Change in bite or occlusion

- Pain or tenderness

- Denture no longer fits

- Loose tooth or teeth

- Restriction of tongue or jaw movements

- Taste change

- Paraesthesia

- Chronic cough

- Dry mouth

- Speech changes

- Color change

- Symptoms related to primary tumor elsewhere

Researchers advocate the use of comprehensive oral examination (COE) as the "gold standard" for early detection. ${ }^{6}$

\section{SCREENING TOOLS FOR ORAL CANCER}

- Visual inspection

- Self- Examination

- Toluidine blue

- Fluorescence imaging

- Exfoliate cytology/Brush biopsy

- Saliva Examination

\section{SCREENING PROTOCOL}

Early detection of oral cancer and related premalignancy requires an appropriate screening and diagnosis protocol. It has been recommended that all adult patients 18 and over be screened annually, ${ }^{7}$ even if medical and dental histories elicit no risk factors. Known-risk patients should be screened every six months.

The screening protocol should include medical and dental history, unaided and aided visual examination, and palpation. All oral structures must be thoroughly examined, and any abnormalities should be recorded on a mouth map. If suspicious lesions are found during the screening procedure, the lesion must be biopsied or the patient referred to a specialist for further evaluation.

\section{BIOPSY PROTOCOL}

The two basic biopsy techniques for definitive diagnosis of oral mucosal lesions are incisional biopsy and excisional biopsy. The brush biopsy (CDx) is a third type of biopsy that can be used as a preliminary diagnostic tool. The brush biopsyuses a stiff brush to obtain a full-thickness sampling of epithelial cells for examination, in patients with mucosal lesions.

The brush biopsy may also be useful as an intermittent preliminary diagnostic technique in patients under observation. ${ }^{8}$ While brush biopsies are practical, simple to perform, and less invasive than an incisional or excisional biopsy, they are insufficient to provide a definitive diagnosis. Incisional or excisional biopsy is the standard-ofcare for definitive diagnosis.

\section{CURRENT TOPICS IN CANCER SCREENING}

TOLUIDINE BLUE: Toluidine blue is a vital stain that binds to nuclear material and preferentially stains tissues with high rates of cellular proliferation.

Toluidine blue can be associated with a high false positive and high false negative rate. For example, $50 \%$ of oral lichen planus lesions were positive and only $42 \%$ of dysplasias stained positively. ${ }^{9}$ Therefore, the provider must be careful not to overextend the utility of this tool. Toluidine blue has also been proposed as a tool to predict progression of oral dysplasia to cancer. In one study, toluidine blue stained lesions with high-risk histologic features, with staining correlated to patient outcome. ${ }^{10}$ There is no evidence to support the use of toluidine blue as an oral cancer screening tool for the general population

TISSUE FLUORESCENCE: Certain cellular molecules, especially those within mitochondria and lysosomes, absorb the energy from light of specific wavelength. When these molecules move back to their unexcited state, the absorbed energy is released. This energy is referred to as fluorescence emissions. Porphyrins in erythrocytes also contribute to autofluorescence. Oral cancer cells have different autofluorescence emission relative to 
normal oral mucosa. Technology, such as VELscope, has been developed to capitalize on this difference in autofluorescence between cancer and normal tissue and to use this approach to detect pathologic lesions in the oral cavity.

TISSUE REFLECTANCE: Chemiluminescence, or tissue reflectance, is an adjunctive screening tool that is used to detect cervical premalignant or malignant lesions. Two systems using chemiluminescence developed for the oral cavity are ViziLite $^{\circledast}$ Plus and MicroLux DL. The increased nuclear to cytoplasmic ratio characteristic of squamous cell carcinoma increases light reflectance relative to normal epithelium. The sensitivity of the chemiluminescence devices for highlighting potentially pathologic lesions is high; however, benign lesions, such as leukoedema and traumatic ulcers, test positive examination under standard incandescent lighting. Oh and Laskin reported that the use of ViziLite ${ }^{\circledast}$ actually made visualizing lesions more difficult due to the distracting highlights it created. At best, tissue reflectance technology can be used as an adjunctive screening tool to the conventional oral examination. A scalpel biopsy of suspicious lesions is required.

BRUSH CYTOLOGY: The brush biopsy (Oral CDx ${ }^{\circledast}$ from CDx Laboratories) is intended for oral lesions that appear innocuous and would not normally be biopsied by the provider. The brush biopsy is intended to be an adjunct diagnostic tool and not a screening tool. Demonstrating efficacy for the diagnosis of suspicious oral lesions with brush cytology is not easy. The population investigated must have lesions that are not already highly suspicious for malignancy, and all lesions in the population must be subjected to surgical biopsy.

GENOMICS: The human genome project, completed in 2002, was to revolutionize surgery and medicine. Scientists predicted that once the entire human genome sequence was known that many cancers, including oral cancer, would be curable.
However, our comprehensive understanding of the human genome has not cured cancer

\section{CONCLUSION}

The ability to identify lesions and to predict which lesions will undergo malignant transformation would facilitate early diagnosis and subsequent disease management tailored to the individual patient. The ultimate goals are to reduce both mortality and morbidity, and to improve patients' quality of life.

\section{REFERENCES}

1. Lingen MW, Kalmar JR, Karrison T, Speight PM. Critical evaluation of diagnostic aids for the detection of oral cancer. Oral Oncol 2008; 44(1):10-22.

2. American Cancer Society. Oral Cancer. Available at: http://www.

cancer.org/acs/groups/content/@nho/documents/docum ent/oralcancerpdf.pdf. Accessed July 2, 2018.

3. Oral Cancer Statistics. Oral Cancer in the United States. Available at: http://www.nidcr.nih.gov/FactSheet2.pdf. Accessed July 2, 2018.

4. The Oral Cancer Foundation web site. Oral Cancer Facts. Available at: http://oralcancerfoundation.org/facts/index/htm. Accessed June 28, 2018.

5. Kammer C. Shine a light on oral cancer. Dental Products Report. October, 2009. Available at: http://www.dentalprodcutsreport.com/ articles/show/dprloog. Accessed May 3, 2018.

6. Rethman MP, Carpenter W, Cohen EEW, Epstein J, et al. Evidence based clinical recommendations regarding screening for oral squamous cell carcinomas. J Am Dent Assoc 2010; 141(5):509-20.

7. Joseph BK, Oral cancer: prevention and detection, Med Princ Pract. 2002; 11(18):32-5.

8. Kosicki DM, Riva C, Pajarola GF, Burkhardt A, Gratz KW. OralCDx brush biopsy - a tool for early diagnosis of oral squamous cell carcinoma, Schweiz Monatsschr Zahnmed, 2007; 117(3).222-7.

9. Martin IC, Kerawala CJ, Reed M. The application of toluidine blue as a diagnostic adjunct in the detection of epithelial dysplasia. Oral Surg Oral Med Oral Pathol Oral Radiol Endod. 1998; 85(4):444-6.

10. Zhang L, Williams M, Poh CF, et al. Toluidine blue staining identifies high-risk primary oral premalignant lesions with poor outcome. Cancer Res. 2005; 65(17):8017-21. 